\title{
Factors influencing perceived angular velocity
}

\author{
MARY K. KAISER \\ NASA Ames Research Center, Moffett Field, California \\ and \\ JACK B. CALDERONE \\ San Jose State University, San Jose, California
}

\begin{abstract}
The assumption that humans are able to perceive and process angular kinematics is critical to many structure-from-motion and optical flow models. The current studies investigate this sensitivity, and examine several factors likely to influence angular velocity perception. In particular, three factors are considered: (1) the extent to which perceived angular velocity is determined by edge transitions of surface elements, (2) the extent to which angular velocity estimates are influenced by instantaneous linear velocities of surface elements, and (3) whether element-velocity effects are related to three-dimensional (3-D) tangential velocities or to two-dimensional (2-D) image velocities. Edge-transition rate biased angular velocity estimates only when edges were highly salient. Element velocities influenced perceived angular velocity; this bias was related to 2-D image velocity rather than 3-D tangential velocity. Despite these biases, however, judgments were most strongly determined by the true angular velocity. Sensitivity to this higher order motion parameter was surprisingly good, for rotations both in depth ( $y$-axis) and parallel to the line of sight (z-axis).
\end{abstract}

Several optical flow models (Koenderink \& van Doorn, 1975, 1976; Lappin, 1990) and structure-from-motion (SFM) models (Braunstein, Hoffman, \& Pollick, 1990; Ullman, 1979) assume a competence for processing angular kinematics, particularly the extent and velocity of rotations. Optical flow models are often concerned with rotations about the line-of-sight axis (i.e., $z$-axis rotations or rotary motion), since these rotations constitute the curl component of the motion decomposition. SFM models, on the other hand, deal with the recovery of spatial information from rotations about any axis not parallel to the line of sight. Presumably, these two classes of rotations rely on different early vision receptors, with the properties of rotary motion receptors understood less well than those of linear motion receptors (Saito et al., 1986; Sakata, Shibutani, Ito, \& Tsurugai, 1986; Tanaka, Fukada, \& Saito, 1989; Tanaka \& Saito, 1989). Despite this difference in early level processing, both classes belong to the same object motion category. Thus, it is useful to investigate the psychophysics of sensitivity to angular velocity across classes of rotational axes.

There is a scarcity of psychophysical data on this competence. The studies that do exist are focused exclusively on either one or the other class of rotational motion. Werkhoven and Koenderink (1991) have conducted the most

We thank Larry Beck and Felix Shung of Sterling Software for their programming support. Walter Johnson, Lyn Mowafy, Peter Werkhoven, and two anonymous reviewers provided helpful comments on earlier versions of this manuscript. Requests for reprints should be sent to Mary K. Kaiser, NASA Ames Research Center, Mail Stop 262-3, Moffett Field, CA 94035-1000. systematic examination of velocity discrimination for rotary motion. They examined ways in which such factors as exposure time and frame duration, pattern density and distribution, spatial scale, and angular velocity influence discrimination thresholds; they proposed a model which suggests that the estimation of rotary motion is mediated by local estimations of linear velocity. The effects of visual texture (Walker, 1975) and spatial frequency (Campbell \& Maffei, 1979) on perceived rotary velocity have been examined as well. Walker (1975) reported a positive monotonic relation between subjective velocity and the coarseness (or size) of visual texture. Campbell \& Maffei, using a 1-cycle-per-degree (cpd) reference, noted an increase in apparent velocity as spatial frequency was increased, peaking at $4 \mathrm{cpd}$; at higher spatial frequencies, apparent velocity decreased. However, the velocity used by Campbell and Maffei (1979) was so low $\left(6^{\circ} / \mathrm{sec}\right)$ that observers may have been judging the change in orientation over time rather than the angular velocity. Kaiser (1990) studied angular velocity discrimination for rotations of objects in depth and found that perceived velocity was influenced by object size and edge-transition rate. Cooper, Gibson, Mowafy, and Tataryn (1987) investigated people's ability to extrapolate rotations in depth, but they did not examine angular velocity perception per se.

In the present experiments, we examined angular velocity perception for rotations both in depth and in the image plane and explored the influence of several object properties on this motion parameter. Although angular velocity is a primitive construct within the kinematic domain, it must be construed as a higher order parameter within the optical domain. An object's angular velocity 
is not specified by any simple optical variable; rather, its recovery requires the integration of multiple element velocities scaled to object dimensions (Kaiser, 1990).

Two principal object properties were examined: (1) texture density (for rotations in depth, this factor determines the rate of edge transitions-i.e., the number of texture elements that pass an object's boundary per unit of time), and (2) object size, which determines the tangential linear velocities (and two-dimensional [2-D] image velocities) of texture elements for a given angular velocity. Texture density was manipulated in the first experiment, utilizing both random and regular texture distributions; object size was manipulated in the second experiment. In a final experiment, different regions of the image were occluded to determine whether element velocity effects were linked to three-dimensional (3-D) tangential velocities or 2-D image velocities.

\section{EXPERIMENT 1}

The first experiment examined whether observers' perceived angular velocity was affected by the rate at which texture elements transit an object's boundaries, or edges. Research on the perception of linear motion indicates that edge rate can significantly influence velocity estimates (Denton, 1980); the greater the number of edge transitions, the faster the perceived speed.

Previous work on perceived angular velocity demonstrated an edge-transition-rate effect (Kaiser, 1990). However, since solid forms were used as stimuli in that study, edge rate was confounded with boundary-transformation rate; forms with more face transitions per rotation also had higher boundary-transformation rates. Thus point light spheres (whose boundaries do not transform under rotation) were used in the present experiment to allow a more direct examination of the effect of texture-element density and edge-transition rate per se.

The stimulus spheres used in this experiment were composed of texture elements distributed in either random or regular patterns. For rotations about the $y$-axis, spheres with regularly distributed patterns have highly salient edge transitions, whereas the edge transitions for spheres with random elements are more subtle. In rotations about the $z$-axis, texture elements do not undergo edge transitions. Thus, the effect of texture density could be investigated independent of edge-transition rate.

\section{Method}

Observers. Eight observers ( 4 males and 4 females) participated in this and in the following two experiments. Their ages ranged from 19 to 25 years. All had normal or corrected-to-normal binocular vision. Prior to participation, each observer was informed of the general nature of the research. The order in which the experiments were completed was randomized across observers to avoid systematic practice effects.

Apparatus and Stimuli. The experimental program was run on a Silicon Graphics Personal IRIS 4D/25TG workstation. Stimuli were displayed on a 1,280 $\times 1,024$ pixel, $48.25-\mathrm{cm}$ (diagonal) RGB monitor with a $60-\mathrm{Hz}$, noninterlaced raster refresh rate. Displays were updated at 30 frames/sec. Angular velocity was varied by altering the degree of angular displacement between frames. The viewing geometry used in the experiments is shown in Figure 1 . At the prescribed viewing distance of $1 \mathrm{~m}$, the entire display window subtended approximately $15^{\circ}$ of visual angle. The two stimulus spheres were displayed side by side. Each sphere subtended approximately $4.5^{\circ}$. The observers viewed the display screen binocularly. The experiment was conducted with minimal ambient lighting.

The stimulus spheres used in Experiment 1 are depicted in Figure 2. The stimuli were composed of either 200 or 400 singlepixel white texture elements against a blue background. (A colored background was used to mask motion streaks from phosphor persistence of the points.) The texture elements were either randomly distributed on the surface of the sphere or regularly spaced along longitudinal lines (10 for the 200 -element form, 20 for the 400 -

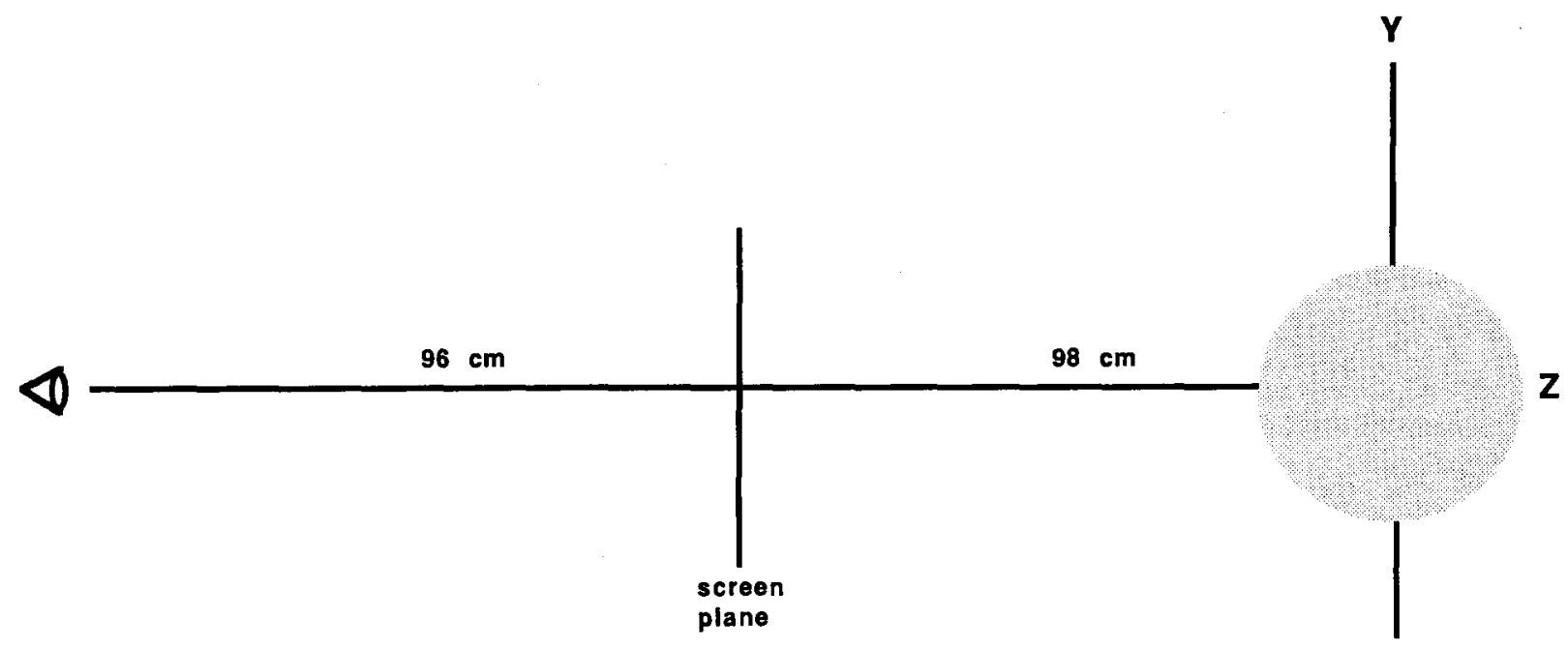

Figure 1. Viewing geometry employed in Experiments 1, 2, and 3. System is shown along the $x$-axis, with the stimulus sphere depicted (not to scale). 

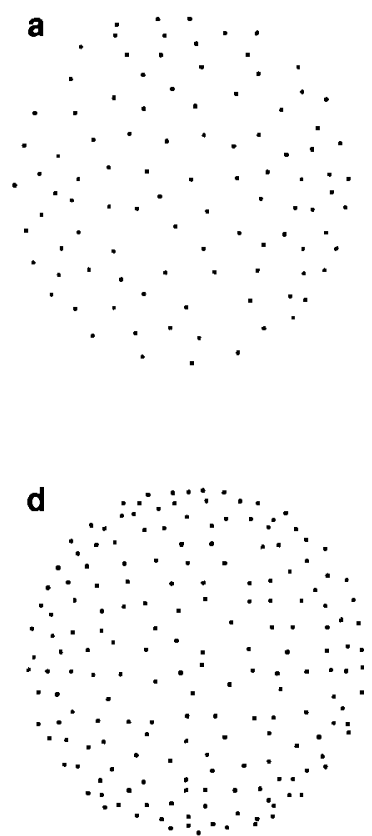
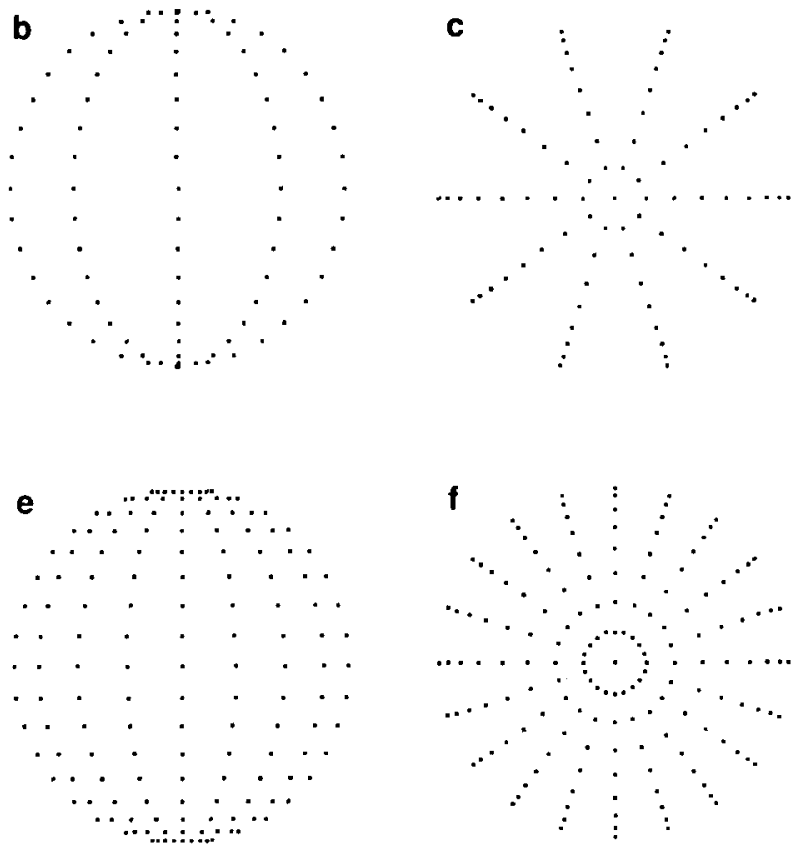

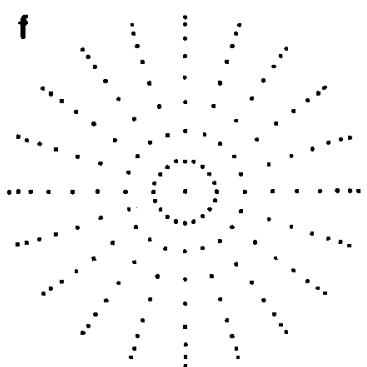

Figure 2. Schematic of stimulus sphere used in the experiments: (a) 200 randomly distributed elements; (b) 200 regularly distributed elements, $y$-axis configuration; (c) 200 regularly distributed elements, $z$-axis configuration, (d-f) 400 element spheres. All spheres were composed of single-pixel elements.

element form). For $z$-axis rotations, the regular forms were rotated $90^{\circ}$ so that the longitude lines formed radial spokes, as shown in Figures $2 \mathrm{c}$ and $2 \mathrm{f}$. The sphere projected $4.5^{\circ}$ in diameter. The 400 element sphere was designated the standard and rotated at $75 \% \mathrm{sec}$ $\left(2.5^{\circ} /\right.$ frame $)$ in all conditions. For the stimuli with regularly distributed elements, rotations occurred with the standard and comparison spheres initially $45^{\circ}$ out of phase. Each stimulus pair consisted of a comparison sphere with 200 texture elements and the standard sphere with 400 texture elements. Spheres rotated about axes either in the picture plane ( $y-y$ axes) or orthogonal to it ( $z-z$ axes).

Design. A within-subjects design was used, with texture-element distribution (random, regular) and rotational axes $(y-y, z-z)$ as experimental factors. Since elements undergo edge transitions in only $y$-axis rotations, the $z-z$ comparison can be viewed as a control for the effect of element density.

Observers were asked to judge relative angular velocities for each stimulus pair. Transformed up-down staircases with decreasing step sizes (Levitt, 1971) were used to track observers' $X_{\mathbf{2 9 . 3}}$ and $X_{\mathbf{7 0 . 7}}$ (i.e., the velocities at which the comparison stimulus was judged faster $29.3 \%$ and $70.7 \%$ of the time) for each condition. Staircases were terminated after eight reversals, with a terminal step size of $1.5^{\circ} / \mathrm{sec}\left(0.05^{\circ} / \mathrm{frame}\right)$. The eight staircases (2 texture element distributions $\times 2$ rotational axes $\times 2$ tracking points) were interlaced to ensure independence of responses for adjacent trials of each staircase.

Procedure. Observers were seated at the correct viewing position for the projection geometry, although head and eye position were not constrained. On each trial, an auditory warning sounded, and the standard and comparison stimuli were displayed simultaneously for $4 \mathrm{sec}$. At the termination of the display sequence, a response menu was highlighted; observers indicated which of the two spheres appeared to rotate at a faster rate. The position (left vs. right) of the 400 -element standard varied from trial to trial. Observers were not given feedback during the experiment. About $45 \mathrm{~min}$ were required for an observer to complete Experiment 1.

\section{Results}

For each condition, $X_{29.3}$ and $X_{70.7}$ were determined for observers by taking the mean of their final six midrun estimates. The difference of these two points is equal to $1.09 \sigma$, so a multiplier of 0.619 was used to obtain an estimate of the differential threshold (DT). The average of these two points was used to estimate each observer's point of subjective equality (PSE). (The latter estimation procedure requires a symmetry assumption for the underlying psychometric function.)

Averaged across observers, DTs ranged from $4 \%$ to $6 \%$. There was no significant effect for element distribution or rotational axis. The PSE data are presented in Table 1. PSEs differed significantly from the point of objective equality (POE) only for the $y-y$ rotational axes with regular element distributions $[F(1,7)=31.92, p<.01]$. In this condition, the sphere with fewer texture elements had to rotate $13 \%$ faster, on average, to have the same perceived angular velocity. No effect for the number of texture elements was noted for $z-z$ axes' rotations in either the regular- or the random-distribution conditions.

These results suggest that the edge-transition rate of the texture elements can influence perceived velocity if the configuration of the elements makes these transitions 
Table 1

Point of Subjective Equality (PSE) Data for Experiment 1

\begin{tabular}{lcc}
\hline Element Distribution & Regular & Random \\
\hline & $y-y$ Axes & \\
(PSE-POE)/POE & .13 & .01 \\
$F(1,7)$ & $31.92 \dagger$ & 1.00 \\
& $z-z$ Axes & \\
(PSE-POE)/POE & .02 & .02 \\
$F(1,7)$ & 0.83 & 0.69 \\
\hline
\end{tabular}

Note-PSEs can be recovered by converting the ratios above as follows: $\mathrm{PSE}=($ ratio +1$) \cdot 75^{\circ} / \mathrm{sec}$.

salient. The magnitude of this bias, however, is smaller than would be expected if the observer was equating edge rate (i.e., $100 \%$ for the stimuli used). Further, no effect was noted for element density in the absence of edge transitions (i.e., the $z-z$ axes condition). This suggests that the temporal frequency of edge transitions, not the density of the texture elements per se, influences perceived rotational velocity.

\section{EXPERIMENT 2}

Experiment 2 examined the influence of the linear velocity of elements on perceived angular velocity. Werkhoven and Koenderink (1991) proposed that rotary velocity estimates are mediated by estimates of the local linear velocity of texture elements. They investigated this hypothesis in the context of velocity discrimination thresholds and biases in perceived velocity (Werkhoven, 1990) for rotary motions. However, they did not investigate whether such mediation occurs for rotations in depth. In Experiment 2, the effect of local linear velocities was examined by manipulating the relative size of the comparison sphere. Local linear velocities of the texture elements increase as a function of the sphere's size ( $v_{\text {linear }}=2 \pi r$, where $r$ is the distance from the element to the axis of rotation). Thus, the local linear velocities of texture elements on a comparison sphere twice the diameter of the reference sphere were twice those of corresponding elements on the reference sphere.

Kaiser (1990) found a velocity bias related to size for the rotation of solid objects in depth. The current experi-

Table 2

Point of Subjective Equality (PSE) Data for Experiment 2

\begin{tabular}{lcc}
\hline Element Distribution & Regular & Random \\
\hline & $y-y$ Axes & \\
(PSE-POE)/POE & .17 & .12 \\
$F(1,7)$ & $9.36^{*}$ & $8.18^{*}$ \\
& $y-z$ Axes & \\
(PSE-POE)/POE & .14 & .07 \\
$F(1,7)$ & $20.52 \dagger$ & $6.97^{*}$ \\
& $z-z$ Axes & \\
(PSE-POE)/POE & .05 & .12 \\
$F(1,7)$ & $3.80^{*}$ & $32.80 \dagger$ \\
\hline
\end{tabular}

Note-PSEs can be recovered by converting the ratios above as follows: PSE $=($ ratio +1$) \cdot 75^{\circ} / \mathrm{sec} .{ }^{*} p<.05 . \quad \dagger p<.01$. ment extends this work by examining rotations both in depth and within the picture plane. Also, the use of texture elements in the present study clearly specifies that stimulus objects are of different sizes at the same distance. In the Kaiser study, observers could have misinterpreted the smooth-surfaced stimuli as equal-sized objects at different distances (the geometric distortions resulting from such a misinterpretation were subtle).

\section{Method}

Except for the differences described below, the method was the same as that in Experiment 1.

Observers. The same observers participated in all experiments. The order of participation was randomized across observers.

Apparatus and Stimuli. Two 400 -element spheres were shown in each stimulus pair. The standard sphere was the size used in Experiment $1\left(4.5^{\circ}\right.$ of visual angle). The comparison sphere was half that size (i.e., $2.25^{\circ}$ of visual angle). The size of the texture elements was equivalent on the two spheres ( 1 pixel), suggesting two different-sized objects at the same distance rather than two equalsized objects at near and far distances. All observers reported seeing the stimuli as being of different sizes, not at different distances. Due to the greater computational complexity of the stimuli, the display update rate was reduced to $20 \mathrm{~Hz}$. The angular velocity $(\omega)$ of the standard was kept at $75 \% / \mathrm{sec}$ by increasing the interframe angular displacement to $3.75 \%$ frame.

Texture elements were either randomly or regularly distributed on the sphere's surface, as described for the previous experiment and shown in Figure 2. Observers were asked to make relative velocity judgments for pairs of spheres rotating about parallel $(y-y$ and $z-z$ ) and orthogonal $(y-z)$ axes. For the orthogonal axes stimuli, the $y$-axis sphere was the standard in the randomly distributed condition; the $z$-axis sphere served as the standard in the regularly distributed condition.

Design. A within-observers design was used, with element distribution (random vs. regular) and rotational axes $(y-y, z-z$, or $y-z)$ as experimental factors. The resulting 12 staircases ( 2 element distributions $\times 3$ rotational axes $\times 2$ tracking points) were interleaved as in Experiment 1.

\section{Results}

The data were analyzed as in Experiment 1 . The DT data for Experiment 2 were similar to those of Experiment 1 ; values ranged from $3 \%-6 \%$ with no systematic variation associated with dot distribution or rotational axes. PSEs were significantly elevated relative to POEs for all rotational conditions, indicating that the smaller sphere had to rotate faster to have an equivalent subjective angular velocity (Table 2). However, this bias, while statistically significant, does not suggest that observers were matching element velocities. To do so would require that the smaller sphere rotate at a rate twice that of the larger sphere (i.e., a bias of $100 \%$ ). The largest bias observed was only $17 \%$, in the $y-y$ regular-distribution condition.

\section{EXPERIMENT 3}

Experiment 3 examined the extent to which angular velocity judgment biases caused by element velocity reflects the 3-D tangential velocities of the elements or their 2-D image velocities. Presumably, the bias introduced by the local linear velocities of elements could reflect either mo- 
tion parameter. A bias related to 2-D velocity implies that the contamination of element velocity occurs at a relatively early stage in motion processing-that is, prior to the recovery of 3-D tangential velocities. To investigate this question, we had observers compare angular velocities of two forms, identical in size and surface-texture density, but with different regions of their 2-D images occluded. Thus, for rotations with axes in the picture plane (e.g., $y$-axis rotations), the image velocity of an element varies sinusoidally as it moves across the screen, with maximal velocity as the element passes the midline. In terms of 3-D velocity, an element's velocity has a constant value $(\omega \cdot 2 \pi r)$ throughout the rotation. Three-dimensional velocity does vary, however, as a cosine function of latitude, with maximal velocity at zero latitude (i.e., the equator) and minimum velocity $(v=0)$ at $180^{\circ}$ latitude.

Consider the stimuli depicted in Figure 3. The sphere on the left (3a) has the central region occluded; the one on the right ( $3 \mathrm{~b}$ ) has the side regions occluded. If the two spheres rotate at equal angular velocities, the average 3-D element velocity is somewhat greater in $3 a$, since the higher latitudes are occluded. In terms of 2-D image velocity, the average is greater in $3 \mathrm{~b}$. Thus, an observer's PSEs will reflect whether judgments are based on angular velocity, 3-D element velocities, or 2-D image-element velocities:

1. A PSE at which $\omega_{\text {central occluded }}=\omega_{\text {sides occluded }}$ implies that observers utilize angular velocity.

2. A PSE at which $\omega_{\text {central oceluded }}>\omega_{\text {sides occluded }}$ implies that observers utilize 2-D image-element velocities.

3. A PSE at which $\omega_{\text {central occluded }}<\omega_{\text {sides occluded }}$ implies that observers utilize 3-D element velocities.

\section{Method}

Except as noted, the method was the same as it was in the previous experiments.

Apparatus and Stimuli. The stimuli used were the randomly and regularly distributed 400-dot forms (Figures $2 \mathrm{~d}$ and $2 \mathrm{e}$ ) updated at $20 \mathrm{~Hz}$. An occluding mask was placed over the display screen in such a way that only the central region of the standard sphere and the outer regions of the comparison sphere were visible, as is shown in Figure 3. The regions visible on the two spheres were
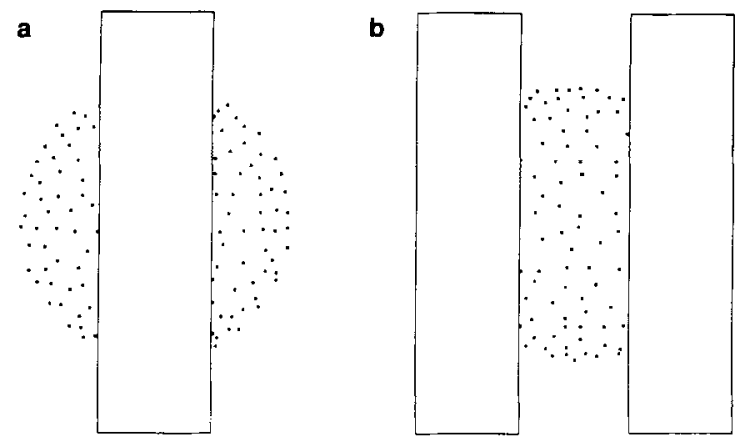

Figure 3. Stimuli used in Experiment 3, with either the central region (3a) or the outer regions (3b) occluded.
Table 3

Point of Subjective Equality (PSE) Data for Experiment 3

\begin{tabular}{lcc}
\hline Element Distribution & Regular & Random \\
\hline & $y-y$ Axes & \\
(PSE-POE)/POE & .06 & .09 \\
$F(1,7)$ & $7.51^{*}$ & $14.40 \dagger$ \\
\hline
\end{tabular}

Note-PSE can be recovered by converting the ratio above as follows: PSE $=($ ratio +1$) \cdot 75 \%$ sec. ${ }^{*} p<.05 . \quad t p<.01$.

equated in 2-D image area. The average 2-D image velocity for the center-occluded sphere was approximately 6 pixels/frame; for the sides-occluded sphere, the average was approximately 3.8 pixels/ frame. Observers were asked to judge relative angular velocities for pairs of spheres with randomly and regularly distributed elements rotating about their $y$-axes.

Design. A within-subjects design was used, with element distribution (random vs. regular) as the principal experimental factor. In order to provide more stable estimates of occlusion effects, two replications of each staircase were used. As in the previous experiments, both $X_{29.3}$ and $X_{70.7}$ staircases were used. Thus, 8 staircases ( 2 element distributions $\times 2$ replications $\times 2$ tracking points) were interleaved for presentation to each observer.

\section{Results}

The data were analyzed as in Experiments 1 and 2. As in Experiments 1 and 2, DTs were not systematically affected by element distribution and were in the range of $4 \%-6 \%$. PSEs, shown in Table 3 , were significantly greater than POE for both the regular- and the randomelement distributions, indicating that the sphere with the central region occluded had to rotate faster to achieve a subjectively equal angular velocity. This would suggest that observers' judgments were influenced by the 2-D image velocities of texture elements rather than 3-D tangential velocities. However, it should be noted that the sphere with the central region occluded would require an angular velocity approximately 1.60 times that of the sidesoccluded sphere for 2-D image velocity to be equated. In the data, the demonstrated bias was only $6 \%$ for the regularly distributed elements and $9 \%$ for the randomly distributed elements. Thus, as before, element velocity biased perceived angular velocity but was not its major determinant.

\section{GENERAL DISCUSSION}

Across the three experiments, observers demonstrated an ability to discriminate as little as $\mathbf{5 \%}$ difference in angular velocity - a somewhat better performance than has previously been reported (e.g., Kaiser, 1990; Werkhoven \& Koenderink, 1991). However, the stimulus forms used in these studies differed from the solid forms used in Kaiser (1990), and they are fairly well optimized along the stimulus dimensions (e.g., frame duration, number of texture elements, size, and angular velocity) investigated by Werkhoven and Koenderink (1991). Perhaps these factors account for the improved discrimination performance. As in earlier studies, performance was surpris- 
ingly robust with respect to rotational conditions (e.g., whether the forms rotate about parallel or orthogonal axes), and it compares remarkably well with thresholds reported for simple linear velocity discrimination (McKee, 1981).

The data from Experiment 1 indicate that edge rate, but not texture density per se, influences perceived angular velocity. Thus, the number of texture elements did not influence perceived velocity for $z$-axis rotations. Walker (1975) reported an effect of texture on perceived rotary velocity. However, he varied stimulus density over a full $\log$ unit range to effect a $25 \%$ bias. Given that our stimulus density varied by only a factor of two, it is not surprising that no effect was evident.

For the $y-y$ axis rotations, in which texture elements underwent edge transitions, the number of elements did affect relative velocity judgments when these edge transitions were made more salient by arranging the elements along meridian lines. ${ }^{1}$ The bias observed in this condition is similar in magnitude to that noted in Kaiser (1990). This suggests that salient edge transitions, even in the absence of contour deformations, influence perceived angular velocity. Thus, as in linear motion, perceived velocity is biased by temporal frequency properties of the stimuli.

No effect of number of elements on discrimination thresholds was noted in either rotational condition. Werkhoven and Koenderink (1991) did find such an effect, but their stimuli had far fewer elements (range: 1-32). In fact, their data suggest that any improvement in discrimination asymptotes at 8 or 16 elements; the present stimuli possessed either 100 or 200 visible elements and thus clearly exceeded the range in which performance improvement was demonstrated.

The results of Experiment 2 indicate that element velocities do bias angular velocity estimates, both for rotations in depth and within the image plane. However, as noted in Kaiser (1990), these biases are sufficiently small to exclude any model that would suggest element-velocity matching. Nonetheless, element velocity does play some role in mediating perceived angular velocities both for rotations in depth and within the picture plane.

The results of Experiment 3 suggest that influence of linear element velocity is related to 2-D image velocity rather than to 3-D tangential velocities for rotations in depth. Again, however, the bias in angular velocity judgments are relatively small, indicating that fairly canonical recovery of true angular velocity is possible.

In fact, in all three of the present experiments, as well as in previous studies (e.g., Kaiser, 1990), the motion parameter accounting for most of the variance in observers' judgments is the true angular velocity, $\omega$. Whereas extraneous spatiotemporal characteristics of the stimuli shift PSEs, these shifts are relatively minor. Further, whereas Werkhoven and Koenderink (1991) found evidence that spatiotemporal characteristics affect discrimination sensitivity, most of the threshold elevation is observed only at extreme values of the stimulus parameters (e.g., frame times longer than $60 \mathrm{msec}$; fewer than 8 texture elements; stimulus forms smaller than $1^{\circ}$ or larger than $10^{\circ}$ of visual angle; angular velocities of $40^{\circ} / \mathrm{sec}$ or less). The influences of these spatiotemporal object properties may thus reflect the remnants of local measurements that are not fully filtered out during the global mediation that leads to the recovery of higher order angular kinematics. As such, they are most apparent for neardegenerate stimulus cases in which global processing is hampered.

Within more moderate values in Werkhoven and Koenderink's (1991) stimulus space, and within the range of stimulus parameters used in the present and in previous (Kaiser, 1990) studies, sensitivity is quite robust; it compares favorably with velocity-discrimination thresholds for simpler, linear motions (McKee, 1981). Overall, then, the human visual system demonstrates a robust competence for processing basic angular kinematics. This ability stands in marked contrast to observers' visual appreciation of angular dynamics (Proffitt, Kaiser, \& Whelan, 1990).

\section{REFERENCES}

Braunstein, M. L.. \& Andersen, G. J. (1984). Shape and depth perception from parallel projections of three-dimensional motion. Journal of Experimental Psychology: Human Perception \& Performance, 10, $749-760$

Braunstein, M. L., Hoffman, D. D., \& Pollick, F. E. (1990). Discriminating rigid from nonrigid motion: Minimum points and views. Perception \& Psychophysics, 47, 205-214.

Campbell, F. W., MafFeI, L. (1979). Stopped visual motion. Narure, $278,192$.

Cooper, L. A. , Gibson, B. S., Mowafy, L., a Tataryn, D. J. (1987). Mental extrapolation of perceptually driven spatial transformations. Bulletin of the Psychonomic Society, 25, 340-341. (Abstract)

Denton, G. G. (1980). The influence of visual pattem on perceived speed. Perception, 9, 393-402.

Kaiser, M. K. (1990). Angular velocity discrimination. Perception \& Psychophysics, 47, 149-156.

KoEnderink, J. J., \& VAN DOORN, A. J. (1975). Invariant properties of the motion parallax field due to the movement of rigid bodies relative to an observer. Optica Acta, 22, 773-791.

KOENDERINK, J. J., \& VAN DOORN, A. J. (1976). Local structure of movement parallax of the plane. Joumal of the Optical Sociery of America, 66, 717-723.

LAPPIN, J. S. (1990). Perceiving the metric structure of environmental objects. In R. Warren \& A. H. Wertheim (Eds.), The perception and control of self-motion (pp. $\star \star \star-\star \star \star$ ). Hillsdale, NJ: Erlbaum. LEVITT, H. (1971). Transformed up-down methods in psychoacoustics. Journal of the Acoustical Society of America, 49, 467-477.

McKeE, S. P. (1981). A local mechanism for differential velocity detection. Vision Research, 21, 491-500.

Proffitt, D. R., Kaiser, M. K., Whelan, S. M. (1990). Understanding wheel dynamics. Cognitive Psychology, 22, 342-373.

SaIto, H., Yukie, M., Tanaka, K., Hikosaka, K., Fukada, Y., a IWAI, E. (1986). Integration of direction signals of image motion in the superior temporal sulcus of the macaque monkey. Joumal of Neuroscience, 6, 145-157.

Sakata, H., Shibutani, H., Ito, Y., a Tsurugai, K. (1986). Parietal cortical neurons responding to rotary movement of visual stimulus in space. Experimental Brain Research, 61, 658-663.

TANaKa, K. FUKadA, Y., a SAITo, H. (1989). Underlying mechanisms of the response specificity of expansion/contraction and rotation cells in the dorsal part of the medial superior temporal area of the Macaque monkey. Journal of Neurophysiology, 62, 642-656. 
TANAKA, K., SAIto, H. (1989). Analysis of motion of the visual field by direction, expansion/contraction, and rotation cells in the dorsal part of the medial superior temporal area of the Macaque monkey. Journal of Neurophysiology, 62, 626-641.

Ullman, S. (1979). The interpretation of visual motion. Cambridge, MA: MIT Press.

WALKER, J. T. (1975). Visual texture as a factor in the apparent velocity of objective motion and motion aftereffects. Perception \& Psychophysics, 18, 175-180.

WERKHOVEN, P. (1990). Visual perception of successive order. Utrecht University.

WERKHOVEN, P., \& KoENDERINK, J. J. (1991). Visual processing of rotary motion. Perception \& Psychophysics, 49, 73-82.

\section{NOTE}

1. The organization of texture elements along meridian lines does reduce the heterogeneity of velocities. Such reductions have been found to reduce the perceived depth in kinetic depth effect displays (Braunstein \& Andersen, 1984). However, all observers reported perceiving the stimuli as spheres in the $y$-axis conditions (some perceived the $z$-axis stimuli as disks). Thus, the perception of sphericity was robust (perhaps aided by contour information), and velocity biases were not likely caused by perceived form deformations.

(Manuscript received March 28, 1991; revision accepted for publication June 27,1991 .) 